\title{
Les concessions à charge de remblais en Polynésie française ou les politiques face à la privatisation
}

Concessions under embankments in French Polynesia or policies facing privatization

\section{Annie Aubanel}

\section{(2) OpenEdition}

\section{Journals}

Édition électronique

URL : http://journals.openedition.org/jso/7639

DOI : $10.4000 /$ jso.7639

ISSN : $1760-7256$

Éditeur

Société des océanistes

Édition imprimée

Date de publication : 31 décembre 2016

Pagination : 273-289

ISSN : 0300-953x

Référence électronique

Annie Aubanel, «Les concessions à charge de remblais en Polynésie française ou les politiques face à la privatisation ", Journal de la Société des Océanistes [En ligne], 142-143 | 2016, mis en ligne le 31 décembre 2018, consulté le 19 avril 2019. URL : http://journals.openedition.org/jso/7639 ; DOI $10.4000 /$ jso. 7639 


\section{Les concessions à charge de remblais en Polynésie française ou les politiques face à la privatisation}

par

Annie AUBANEL ${ }^{*}$

\section{RÉSUMÉ}

Les concessions dites "à charge de remblais" autorisées par les pouvoirs publics permettent aux collectivités et aux particuliers d'utiliser l'espace maritime littoral en le remblayant. Les réglementations d'attribution de ces concessions sont traitées depuis les premiers textes et il n'y a plus actuellement que des dérogations. Les remblais sauvages et donc illicite au regard de la loi peuvent néanmoins être régularisés. Malgré des progrès en vue de mieux gérer ce problème de la privatisation du littoral, il est clair que les faits démontrent l'absence de volonté politique pour faire respecter l'inaliénabilité du domaine maritime. Les concessions bien que temporaires sont en fait définitives et le droit de passage de trois mètres de largeur sur la plage n'est jamais exigé. Les conséquences de ces remblais sont traitées dans le texte. Entre électoralisme et intérêt public, le pouvoir politique n'a pas encore eu le courage de faire appliquer la loi.

Mots-ClÉs : remblais, concessions, privatisation du littoral, réglementation, Polynésie française

Depuis l'ouvrage de Paskoff (1993), « les milieux côtiers exigus, convoités et fragiles" ont fait l'objet de très nombreux travaux pour assurer leur protection ou du moins d'aménagements s'inscrivant dans une gestion intégrée soucieuse de ne pas provoquer de catastrophes. Le trait de côte a été l'objet d'attentions particulières. Il est l'objet d'un enjeu d'intérêt général compte tenu de l'importance du littoral du point de vue démographique, économique, social, environnemental et culturel. Cette limite entre la

\begin{abstract}
Concessions, called 'backfill load', that are authorized by the authorities allow communities and individuals to use backfilled maritime areas for the public interest, or for the interests of riparian owners of the lagoon in order to enlarge their land. These concessions are issued according to regulations that have evolved in recent decades. There are currently no more concessions permitted except under special exemptions. Wild embankments that are therefore illegal in view of the law may nevertheless be regularized. Despite progress towards better management of the coastal privatization problem, there is clear evidence demonstrating a lack of political will to respect the inalienability of the maritime domain. The temporary concessions that are effectively final have never ensured the right of passage - three meters large - to the beach. Between electioneering schedules and public interest, political powers have lacked the courage to enforce these laws in spite of consequences of these embarkments.
\end{abstract}

KeYwords: embankments, concessions, privatization of the coastline, regulatory, French Polynesia

terre et la mer se trouve par ailleurs directement confrontée à l'élévation mondiale du niveau de la mer due au changement climatique. La gestion du trait de côte est devenue un enjeu primordial et une préoccupation qui s'est encore manifestée récemment au plan national (remise du rapport du comité de suivi pour la mise en ouvre de la stratégie nationale de gestion intégrée du trait de côte, le 19 octobre 2015 au ministre de l'Écologie, du Développement durable et de l'Énergie) comme au niveau international (COP 21). 
La Polynésie française compte $4500 \mathrm{~km}$ de linéaire côtier ce qui représente $29 \%$ du linéaire sous juridiction française de la métropole et de l'outre-mer (shom, in Medde, 2014). Les études sur le trait de côte en Polynésie française ont été initiées au début des années 1990 (Aubanel et al., 1991 ; Aubanel, 1993a-b) sur Moorea, île sœur de Tahiti, et ces travaux font état pour 1990 d'un linéaire côtier anthropisé (par opposition à un trait de côte naturel) de $23 \mathrm{~km}$ soit un tiers du rivage de l'île. Entre 1955 et 1986, l'étude révèle que 20 ha ont été remblayés sur la zone frangeant du lagon ce qui représente $0,4 \%$ de la surface de ce dernier. Depuis cette date, les études sur l'évolution du trait de côte se sont multipliées à Moorea (Polti, 2001 ; Benet, 2009 ; Hostein, 2011) comme sur d'autres îles de la Société (Marquet, 1994; Gros, 1995 ; Leille, 2005; Raynal, 2004 ; Lison de Loma, 2007 ; Vieux et al., 2008) et des Tuamotu (Duvat et Salmon, 2015).

L'évolution de la nature du trait de côte et les surfaces remblayées sur les lagons en Polynésie française sont un révélateur de l'anthropisation et de l'urbanisation grandissante qui se développe dans les îles de ce territoire comme en métropole. Les réglementations qui concernent les remblais et les politiques publiques d'aménagement et de conservation affichés par les autorités ont été variables avec le temps. L'objet du présent travail est de les révéler avec leurs conséquences écologique et économique dans le contexte de phénomènes sociaux et de comportement citoyen.

\section{Le domaine public maritime, le trait de côte et les remblais}

L'une des caractéristiques des îles de la Polynésie française est la présence d'un lagon, exception faites des îles de l'archipel des Marquises et de Rapa dans l'archipel des Australes. Cet espace lagonaire est une surface marine protégée des assauts de l'océan par le récif barrière pour les îles hautes et par la couronne récifale émergée et les motu pour les atolls.

"Pour qu'un bien fasse partie du domaine public, il doit être la propriété d'une personne publique et il doit être affecté à l'utilité publique. Le domaine public fait l'objet, par l'administration, d'une délimitation unilatérale, obligatoire, récognitive (déclarative) et non définitive, faite sous réserve des droits des tiers, en application des règles de délimitation. La limite du domaine public maritime naturel est fixée au point où les plus hautes eaux peuvent s'étendre, en l'absence de perturbations météorologiques exceptionnelles ${ }^{1}$. L'incorporation aux rivages de la mer est déterminée par un phénomène naturel qui est le fait qu'une dé- pendance soit alternativement couverte et découverte par le plus grand flot annuel. » (Villot, $1990: 21$ )

La limite du domaine public maritime induit sa mobilité puisqu'elle est liée aux courants, à la sédimentation de certaines zones, à l'accrétion et à l'érosion d'autres. Quand elle est naturelle elle est soumise aux variations des environnements marin, littoral et terrestre, qu'ils soient naturels ou anthropiques. Prenons comme exemple le nettoyage d'un hoa ("chenal») entre deux motu ou le creusement d'une passe dans un atoll, la réalisation d'un remblai ou d'un épis, la construction d'une route ou d'un lotissement, etc. Il n'y a qu'artificiellement qu'on peut espérer la fixer et ce pour une durée variable, limitée dans le temps. Dans ce cas le domaine public maritime cesse d'être naturel pour devenir artificiel.

Hormis à proximité des passes et lors de très mauvais temps, l'espace lagonaire est un plan d'eau dont la tranquillité est semblable à celle d'un lac. Il est donc possible de demander au pays (collectivité de la Polynésie française) qui est le gestionnaire de ce milieu inaliénable et imprescriptible une concession maritime qui est une forme d'utilisation privative. Il existe deux types de concessions maritimes.

La première est demandée pour utiliser un espace lagonaire et un volume d'eau aux fins d'élevage de nacres ou de poissons. Elle ne modifie pas la morphologie du milieu hormis l'installation de quelques infrastructures et mouillages et la pollution engendrée par l'activité qui reste maritime. La demande est motivée par une activité économique comme les parcs à poissons, la collecte de naissains ou l'élevage de nacres.

La seconde doit permettre d'agrandir la surface terrestre d'une propriété littorale en remblayant la partie adjacente du lagon là où la profondeur est modeste. Cette concession est appelée "à charge de remblais " car elle transforme un milieu marin en milieu terrestre. La motivation affichée par les demandeurs est, la plupart du temps, de vouloir lutter contre l'érosion du rivage et son inondation lors de fortes houles. Conjointement à ces arguments les avantages des remblais pour les résidents en bord de mer sont un gain de terrain et la tranquillité par la suppression de tous passages pour les promeneurs. Les zones remblayées se matérialisent au niveau de la ligne de rivage par des remparts de blocs, volcaniques ou plus rarement coralliens, au pied desquels arrive la mer à marée haute, parfois même à marée basse. Mais la construction de mur en béton est la solution la plus commune. Sur ces remblais, et en amont du rempart de blocs ou du bâti en ciment qui borde le lagon, du sable corallien peut être répandu, réalisant ainsi une "plage artificielle " privée à proximité 
du lagon, mais la transformation en jardin est la plus courante.

Dans le contexte des concessions maritimes à charge de remblais, nous aborderons :

- la réglementation mise en place par les gouvernements successifs et sa faiblesse et son applicabilité,

- les études réalisées,

- l'impact de ces remblais sur les plans écologique, économique et social,

- le rôle des services administratifs et leurs limites,

- la politique du gouvernement concernant les remblais.

\section{Les règlementations}

L'espace lagonaire immergé qui s'étend de la ligne de rivage au front océanique du récif barrière pour les îles hautes abrite à la fois des ressources vivantes et inertes exploitées de tout temps par les habitants de ces îles. C'est également un espace de communication et de loisir protégé des assauts de l'océan. Certaines études historiques et anthropologiques nous assurent que cet espace privilégié pouvait être autrefois inclus dans «la propriété privée » d'une famille, sans pour autant que cette dernière puisse interdire la circulation des embarcations au-dessus de cet espace (Bambridge, 2013).

Nous allons aborder chronologiquement les différentes réglementations qui, à des époques particulières de l'histoire de la Polynésie française, ont défini les conditions d'occupation du littoral.

\section{De 1842 à 1880 : le protectorat}

Le régime du protectorat s'applique à la Polynésie française de 1842 à 1880 . La loi du 24 mars 1852 (BOEFO, 1852:314) qui porte sur l'enregistrement des terres met un terme à cette "propriété coutumière maritime " et va imposer la notion de "une terre = un propriétaire ". L'arrêté du 5 novembre 1862 (BOEFO, 1862 : 188) organise le service du cadastre. Enfin le décret du 24 août 1887 (BOEFO, 1887: 390) concerne la délimitation de la propriété foncière. Lors de la création des "Tomite ${ }^{2}$ ", la description insuffisante des terres déclarées reste aujourd'hui source de nombreux litiges. Bambridge (2013 : 52) note que :
" [...] il est paradoxal de constater que la construction de l'État est concomitante de celle de la question foncière qui, en définitive, repose sur un malentendu historique. "

De nombreuses terres vont rester en indivision. Aujourd'hui encore des terres qui n'avaient pas été cadastrées car trop éloignées du rivage et en hauteur ont été vendues et des recours ont été déposés (exemple du lotissement Miri à Punaauia).

Rappelons que la zone des 50 pas du Roi a été instaurée dans l'acte d'annexion des Marquises du $1^{\text {er }}$ mai 1842 . Un arrêté de 1670 précise que :

« les cinquante pas du Roi doivent commencer leur hauteur du lieu où les herbes et arbrisseaux commencent à croître et à continuer à mesurer dudit lieu, jusqu’à la longueur desdits cinquante pas. »

Selon les textes, la valeur des 50 pas correspondent à une distance entre 50 et 81 mètres. Mais cela ne concerne juridiquement que les îles Marquises. Il est important de noter que :

1) la délibération n ${ }^{\circ} 98-39$ APF du 17 avril 1998 précise dans son article $1^{\text {er }}$ que :

"la zone des cinquante mètres des îles Marquises, telle qu'elle ressort des plans établis par le cadastre pour chacune des îles entre 1926 et 1968 fixe de manière intangible la limite des propriétés privées qui lui sont contiguës. "

2) il n'y a pas de récif corallien aux Marquises.

\section{De 1880 à 1946 : Les Établissements français de l'Océanie (EFO)}

Les EFO de 1880 à 1946 dépendent du ministère de la marine (service des colonies) jusqu'en 1894, date de création du ministère des Colonies.

Il faut noter que la reine Pomare, bien qu'ayant gardé après 1842 l'administration et la juridiction entière sur les " Naturels ", la législation mise en place par la France ne conservera aucun aspect de la gestion des anciens Polynésiens. Quant à Pomare V, il déclare le 29 juin 1880 :

" J'ai transféré mes droits à la France ; j'ai réservé les vôtres, c'est-à-dire toutes les garanties de propriété et de liberté dont vous avez joui sous le gouvernement du Protectorat...

Jusqu'en 1935, la demande de concession devait être formulée auprès du receveur des domaines et la décision était prise par le gouverneur après délibération en conseil privé.

2. Cette procédure déclarative mise en place par ce qui n'était pas encore l'autorité coloniale voulait encourager la formalisation d'une propriété foncière privée largement imaginaire car dans l'ensemble les espaces polynésiens étaient constitués d'inextricables usages collectifs claniques et familiaux. Les tomites ont été les premiers actes fonciers polynésiens par déclaration auprès de l'administration. Cependant cette procédure a donné lieu à de nombreux abus et spoliations. 
Lespace lagonaire devient domaine public maritime à partir de la prise de possession coloniale, suite à l'application du code civil en Polynésie française et, plus particulièrement, de l'article 538, par l'assimilation des lagons aux étangs d'eau salée dont la domanialité publique est déterminée par leur " communication directe et naturelle avec la mer».

Une étude du service des domaines rappelle certains principaux généraux (Roucaute, 1953).

«Le domaine public comprend les biens qui, soit par leur nature, soit par la suite de la destination qu'ils ont reçue de l'autorité, servent à l'usage, à la jouissance ou à la protection de tous, et qui ne peuvent devenir, en demeurant ce qu'ils sont, propriété privée. » (Décret du 28 septembre 1926)

\section{L'auteur note enfin que :}

" Il est regrettable que le service des domaines ait, depuis de longues années, contribué à la confusion existante sur la propriété des eaux et des rivages des lagons, en accordant à des particuliers des concessions définitives de parcelles de ce domaine public maritime, alors que l'inaliénabilité et l'imprescriptibilité de ce domaine constituent ses deux qualités intrinsèques les plus essentielles. " (Roucaute, 1953 : 17)

\section{De 1946 à 1976 : Le " Territoire de Polynésie française, territoire d'outre-mer "}

En 1946, dans le cadre de la Constitution de la IV République et de l'Union française, les EFO deviennent un Territoire d'outre-mer (том) et tous les Polynésiens obtiennent le droit de vote limité jusque-là aux seuls Tahitiens devenus citoyens français en 1880.

Avec la loi-cadre sur les Territoires d'outremer, communément appelée Loi Defferre votée le 23 juin 1956 et appliquée le 22 juillet 1957 , ce territoire d'outre-mer devient la Polynésie française. Le décret n57-812 du 22 juillet 1957 portant institution d'un conseil de gouvernement et l'extension des attributions de l'assemblée territoriale va permettre à la Polynésie française de réglementer les occupations du domaine public maritime (DPM). Cet espace lagonaire devient domaine public maritime territorial tout en respectant les droits acquis.

La délibération $n^{\circ} 71-97$ du $1^{\text {er }}$ juillet 1971 est la dernière à accorder des concessions maritimes définitives. Elle approuvait un contrat type de concession maritime (à charge de remblais). La propriété était acquise "dans la mesure et le jour où le domaine public se trouvait soustrait à l'action des eaux par l'effet des travaux prévus par le demandeur de la concession ", c'est-à-dire par la réalisation du remblai. À noter que les concessions étaient définitives. Au bout de cinq ans les travaux devaient être terminés et le remblai restait " insusceptible de transfert entre vifs, sauf entre parents en ligne directe" pendant dix ans (décret $\mathrm{n}^{\circ}$ 57-812 du 22 juillet 1957 article 45 alinéas $\mathrm{d}$ et $\mathrm{g}$ ). La demande était instruite par le service des domaines et de la propriété foncière. La procédure prévoyait une enquête de commodo et incommodo et "l'adhésion" du service des travaux publics et des mines, et de la commission des monuments naturels et des sites. Les concessions étaient accordées par délibération de l'assemblée territoriale.

La continuité, qui existait avant l'époque coloniale entre le milieu terrestre et le milieu marin, est définitivement rompue et la propriété privée clairement instaurée sur le milieu terrestre. Pourtant, au travers de la réalisation de remblais, une confusion va s'installer entre le domaine privé et le domaine public; confusion qui sera entretenue par l'obtention de concessions maritimes définitives pour les propriétaires ayant un terrain en bordure de lagon. En effet, dans les années soixante, l'installation du CEP (Centre d'expérimentation du Pacifique) va nécessiter des travaux très importants sur les atolls mais également à Tahiti. De nombreux engins très performants pour l'époque seront disponibles, facilitant l'élargissement des routes. Afin d'éviter une procédure d'expropriation pour cause d'utilité publique longue et difficile, la partie des propriétés réquisitionnée pour cet élargissement sera largement compensée par l'octroi d'une concession maritime à charge de remblai.

\section{De 1977 à 1984 : L'autonomie de gestion}

La délibération nº78-128 du 3 août 1978 est celle qui va accorder des concessions maritimes temporaires alors que celle de 1971 accordait des concessions maritimes définitives. Elle portait réglementation en matière d'occupation du domaine public. Elle aborde la définition et la constitution du domaine public (lagons et récifs) et l'occupation temporaire à charge de remblais. C'est le premier texte local à portée générale qui affirme les droits que détient le Territoire en application des dispositions de l'article $538 \mathrm{du}$ code civil (Villot, 1990).

Larticle 6 de cette délibération stipule que: "les biens du domaine public sont inaliénables et imprescriptibles ». Le caractère inaliénable des biens du domaine public est donc confirmé.

Larticle $\mathrm{n}^{\circ} 12$ note le titre exceptionnel du remblai, ainsi qu'une durée déterminée de l'autorisation d'occuper le domaine public maritime, éventuellement renouvelable, sans indication de durée maximale; le nombre maximal de renouvellement de la demande n'étant pas précisé. La concession ne peut être accordée sans l'engagement préalable du 
bénéficiaire d'établir et de matérialiser, à ses frais, un passage public en front de mer praticable à tout moment.

L'article $n^{\circ} 14$ définit la procédure qui inclut une enquête publique avant l'instruction de la demande. La motivation obligatoire de tout refus de la commission des monuments naturels et des sites est nécessaire.

L'autorisation est accordée par le conseil de gouvernement avec instruction du dossier par le service des domaines après consultation du service de l'équipement en ce qui concerne les occupations à charge de remblais.

Cette délibération impose, à quelques exceptions près, les mêmes obligations pour la réalisation du remblai que celles figurant dans le contrat type de la délibération de 1971. Elle rappelle qu'une concession sur le DPM est une droit personnel (droits de créance), non un droit réel et qu'elle ne peut remettre en cause le principe d'inaliénabilité. En effet, la concession temporaire est accordée à titre personnel et précaire et est révocable sans indemnité. À son niveau d'ailleurs, la délibération ne pouvait modifier la nature patrimoniale d'un bien domanial, elle ne pouvait qu'en régler la gestion. La délibération n ${ }^{\circ} 85-1106$ du 31 octobre 1985 modifie la délibération précédente du 3 août 1978.

- L'article 14: Au niveau de la procédure, l'enquête publique est supprimée.

- Larticle 15: La durée de la concession est autorisée pour une durée de 30 ans dans le cadre des projets hôteliers et aquacoles. La procédure de déclassement est précisée. Il n'est pas question pour les demandes des particuliers de durée maximale, de sorte que le renouvellement semble possible.

- L'article 16 (nouveau): il prévoit une commission consultative. La délégation à l'environnement créée en août 1985 fait partie de la commission consultative avec les services en charge de l'aménagement et de la mer. En revanche le seul ministre de l'équipement, de l'aménagement, de l'énergie et des mines préside cette commission. Font partis de cette commission : " deux conseillers désignés par l'assemblé territoriale suivant leur circonscription électorale d'origine; le maire ou le maire délégué de la commune associée du lieu concerné ; le chef du service de l'aménagement du territoire; le chef du service des domaines et de l'enregistrement ; le chef du service de la mer et de l'aquaculture ; le chef du service territorial du tourisme et le chef de la délégation à l'environnement." L'avis de la commission des monuments naturels et des sites est maintenu.

\section{À partir de 1984 : Statut d'autonomie interne}

Cette délibération $n^{\circ}$ 2004-34 APF du 12 février 2004 applicable aujourd'hui porte composition et administration du domaine public en Polynésie française. La différence entre les deux délibérations de 1978 modifiée en 1985 et 2004 concerne en premier lieu l'ajout de plusieurs articles dont la fixation des redevances (section 3) et la demande d'occupation temporaire du domaine public à charge de remblais y compris le déclassement aux fins d'aliénation (chapitre II).

La définition domaine public maritime naturel de 2004 qui concerne notre étude, est plus précise que celle de 1978 (modifié 1985) (tab. 1). Par ailleurs :

- L'article 5 confirme que les biens du domaine public sont inaliénables et imprescriptibles.

- Larticle 7 précise l'inaliénabilité et imprescriptibilité du domaine public maritime en cas de concessions.

- L'article 36 confirme l'obligation d'un passage de 3 mètres le long de la façade maritime permettant à tout moment la circulation des piétons.

Les nouveautés concernent :

- L'article 4 : La délimitation du domaine public maritime des rivages de la mer est précisée. Elle est déterminée par la laisse de haute mer en l'absence de perturbations météorologiques exceptionnelles, laisse qui constitue la limite entre le domaine public et les propriétés privées. La délimitation est soumise aux phénomènes naturels des variations du rivage et constitue des gains ou des retraits de la mer.

- Larticle 8 : La durée des concessions qui était de 9 ans et 30 ans selon les cas est modifiée. Elle varie en fonction de la nature et du type de concession. Elle va de 70 ans pour les concessions de service public et les établissements hôteliers à 30 ans pour les opérations de développement économique, enfin à 9 ans pour les particuliers. Mais elles peuvent être renouvelées.

- L'article 39 prévoit le déclassement ${ }^{3}$ des remblais pour aliénation mais uniquement lorsqu'il s'agit d'une activité économique à caractère permanent. La servitude de passage des 3 mètres est conservée.

- Larticle 40 inclut une dérogation pour les particuliers concernant les demandes d'acquisitions de remblais déclassés par les occupants répondants à certaines conditions (résidence principale, occupation depuis plus de 10 ans, paiement à jour des redevances).

La délibération se réfère à des "concessions temporaires à charge de remblais ". En effet, le Pays restant propriétaire de l'espace remblayé, la concession est temporaire pour la personne qui a demandé la concession et qui en jouit, hormis le cas où l'aliénation la rend propriétaire d'un 


\begin{tabular}{|l|l|}
\hline \multicolumn{1}{|c|}{ Domaine public maritime } \\
\hline \multicolumn{1}{|c|}{ dération de $\mathbf{1 9 7 8}$} & \multicolumn{1}{c|}{ délibération de 2004 } \\
\hline - Des rades & - Des rivages de la mer \\
- Des golfes & - Des lais et relais de mer \\
- Des baies et détroits de peu d'étendue & - Des étangs salés communiquant librement ou par infiltration \\
- Des lagons & ou par immersion de la mer \\
- Des étangs salés communiquant & - Du sol et du sous-sol des eaux intérieures dont les havres, les \\
librement ou par infiltration ou par & rades non aménagés \\
immersion de la mer & - Les lagons jusquà la laisse de basse mer sur le récif côté large \\
- Des rivages de la mer & - Du sol et du sous-sol des golfes, baies et détroits de peu \\
- Des plages & d'étendue \\
- Des lagons et récifs & - Du sol et du sous-sol des eaux territoriales. \\
\hline
\end{tabular}

TABLEAU 1. - Comparaison des définitions du domaine public maritime selon les délibérations de 1978 (modifié 1985) et 2004

domaine public. En revanche, la transformation d'un milieu marin en milieu terrestre est définitive puisqu’à ce jour aucune remise en état n'a eu lieu.

Il reste l'ambiguïté de la nature juridique du remblai réalisé dans le cadre d'une concession temporaire (ce qui rappelle le principe d'inaliénabilité et le caractère personnel du droit de concession) en faisant basculer les remblais du domaine public naturel dans le domaine public maritime artificiel ${ }^{4}$. Se pose alors la question du lien de l'aménagement avec le service public et la nature d'ouvrage public de ces remblais. Sur la base de ces deux questions de nombreux contentieux pourraient voir le jour quant à l'usage et au fondement de ces concessions.

En conclusion, la présence, dans de nombreuses îles hautes, d'un espace lagonaire protégé par un récif barrière, d'un récif frangeant de faible profondeur et de l'étroitesse de la plaine littorale, a incité les propriétaires à étendre leur domaine terrestre par la réalisation de remblais. La réglementation avant 1978 accordait des concessions maritimes à charge de remblais définitive ce qui accentue la confusion entre la notion de propriété avant et après la période coloniale. Chacun retenant ce qui est le plus avantage pour son cas particulier. L'application peu rigoureuse de la réglementation et l'absence d'une politique publique affirmée entretiennent, s'il est nécessaire, cette confusion où l'intérêt public est oublié.

\section{Les études}

Les lignes de rivage occupées par des remblais et leur importance sur le linéaire côtier ont été repérées et quantifiées dans plusieurs études et sur plusieurs îles hautes dont le but était d'établir au fil du temps une anthropisation croissante du littoral. Pour cela des catégories avaient été établies pour décrire la ligne de rivage :

- rochers et cailloutis en position naturelle;

- plages de sable blanc;

- plage de sable noir ;

- zone herbacée avec éventuellement des palétuviers ;

- zone sablo-vaseux détritique à végétation haute ;

- conglomérat ou beach rock;

- embouchures de rivière ;

- murets et rochers mis en place par l'homme.

La première étude à ce sujet a été réalisée sur Moorea (Aubanel, 1993a), étude qui a été renouvelée ultérieurement (Polti, 2001 ; Benet, 2009 ; Hostein, 2011) montrant la dynamique d'une anthropisation croissante du littoral. Par ailleurs une telle démarche a été appliquée sur d'autres îles de la Société (Marquet, 1994 ; Gros, 1995 ; Leille, 2005 ; Raynal, 2004 ; Lison de Loma, 2007) et des Tuamotu (Duvat et Salmon, 2015). On notera que l'intérêt de ces études initiées par les organismes de recherche a été retenu par l'administration qui en commandera certaines (Lison de Loma, 2007 ; Benet, 2009).

Au-delà de l'affichage quantitatif du linéaire côtier occupé par des remblais, dans les îles concernées, certaines études ont évaluées les surfaces remblayées. De telles études ne concernent que Moorea (Aubanel, 1993a-1993b) et un secteur de Tahiti (Gros, 1995). Ce sont ces études qui donnent un aperçu des surfaces aliénées du domaine public maritime au profit de l'intérêt public ou de particulier.

\section{Le cas de la commune d'Arue sur l'île de Tahiti}

L'étude concerne le littoral de la commune d'Arue sur l'île de Tahiti que l'on peut diviser en deux parties, suivant la présence ou non d'un lagon :

4. Sont inclus dans la composition du domaine public maritime artificiel : «Les ports avec leurs dépendances, notamment les digues, môles, jetées, quais, terre-pleins et terrains compris dans l'enceinte des ports, bassins et bassins de radoub, estacades et ducs d'Albe, ainsi que les ouvrages établis dans l'intérêt de la navigation maritime, phares, fanaux, sémaphores et feux flottants, balises, bouées et amers » ainsi que « Les aménagements de littoral réalisés sur le domaine public maritime, notamment, les plages artificielles et les remblais ». 


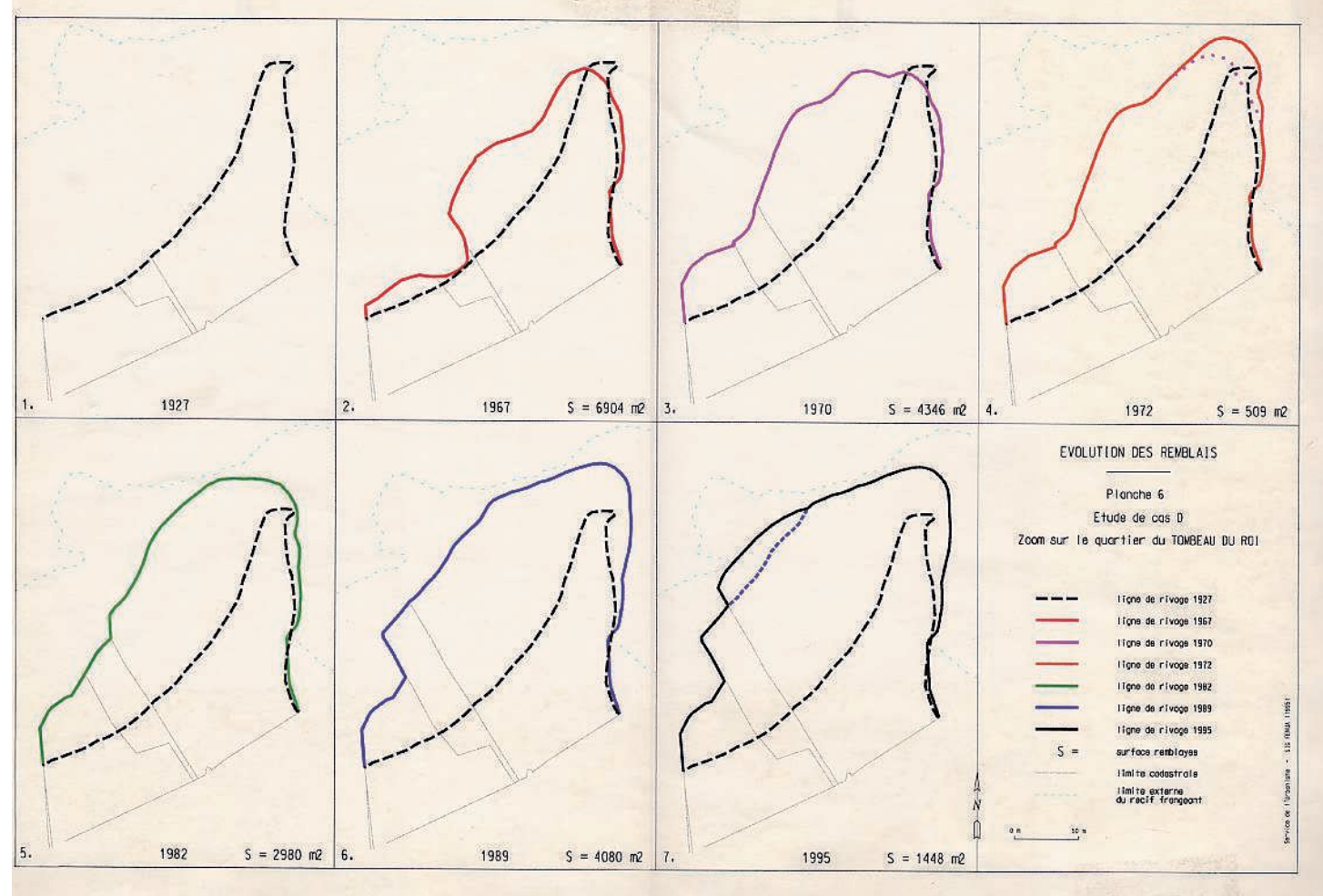

Figure 1. - Évolution des remblais dans un quartier littoral de la commune de Arue, Tahiti de 1927 à 1995 (Gros, 1995)

- La partie est, comprise entre le tombeau du Roi et la pointe du Taharaa protégé de la houle par des hauts fonds sans récif barrière. Cette côte n'est pas remblayée, hormis une portion du récif frangeant de Ahutoru, partiellement protégé par le récif barrière d'Arue

- La partie ouest, comprise entre la rivière Nahoata et la pointe du Tombeau du Roi, protégée par un récif barrière.

Soulignons que l'étude a été menée sur l'ensemble de la commune et qu'elle débute en 1927 grâce à l'exploitation du cadastre de l'époque. On peut considérer que la ligne de rivage de 1927 était quasi naturelle. Au total sur ce linéaire côtier d'environ $4500 \mathrm{~m}$, c'est une surface totale remblayée de $99645 \mathrm{~m}^{2}$ qui a été réalisée et dont la répartition est donnée dans la tableau 2.

L'auteur définit trois catégories de remblais :

- les remblais publics appartenant au Territoire et qui servent à la collectivité ;

- les remblais collectifs appartiennent à des propriétaires privés mais servent la collectivité ;

TABLEAU 2. - Répartition des surfaces remblayées sur l'ensemble du littoral de la commune de Arue (île de Tahiti) selon 3 catégories liées au statut du propriétaire et à l'utilisation du remblai.

\begin{tabular}{|c|c|c|}
\hline $\begin{array}{c}\text { Remblais } \\
\text { publics }\end{array}$ & $\begin{array}{c}\text { Remblais } \\
\text { collectifs }\end{array}$ & $\begin{array}{c}\text { Remblais } \\
\text { privés }\end{array}$ \\
\hline $27438 \mathrm{~m}^{2}$ & $20207 \mathrm{~m}^{2}$ & $52000 \mathrm{~m}^{2}$ \\
\hline
\end{tabular}

- les remblais privés appartenant à des propriétaires privés pour leur seul usage. À noter que plus de la moitié des remblais sont privés.

La figure 1 résume l'évolution de la ligne de rivage dans la partie ouest de la commune de Arue appelé « le tombeau du Roi » car Pomare V y est enterré.

La progression des remblais réalisés sur la mer sans souci particulier d'aménagement harmonieux d'une ligne de rivage, qu'ils soient publics ou privés, autorisés ou non est notable dans ce cas d'étude sur Tahiti. La réalisation de plusieurs études de catégorisation de la ligne de rivage sur l'île de Moorea à des périodes différentes (1993, 2001, 2009) ont mis en évidence l'anthropisation croissante de la ligne de rivage et l'augmentation conséquente des littoraux bordés par des remparts de blocs ou des murs en béton témoins de remblais.

\section{Le cas particulier de Moorea}

Lors de l'élaboration du PGEM (Plan de Gestion de l'Éspace Maritime) de Moorea, les deux principales demandes venant des associations de protection de l'environnement et des pêcheurs concernaient la construction de bungalows sur l'eau (qui nécessitent des concessions maritimes) et la réalisation de remblais. Cette dernière demande s'est traduite dans le règlement du PGEM par l'article 29: 
"Les concessions maritimes à charge de remblais sont interdites sauf pour cause d'utilité publique. Le ré-engraissement des plages artificielles n'est pas concerné par cette interdiction. "

Malgré cet arrêté les demandes de concessions maritimes à charge de remblais sur l'île de Moorea sont réceptionnées par la direction des affaires foncières pour instruction.

Plusieurs évaluations peuvent être rapportées sur le nombre de concessions maritimes à charge de remblais et les surfaces concernées :

a) Suite à la consultation des registres du service des domaines et à la digitalisation de photos aériennes de 1955 au $1 / 40000^{\text {ème }}$ (IGN) ainsi qu'à celles de 1986 au 1/10 000 ${ }^{\text {ème }}$ (SAU), Aubanel (1993), obtient deux évaluations de la surface des concessions maritimes à charge de remblais sur l'île de Moorea respectivement de $98512 \mathrm{~m}^{2}$ et $193510 \mathrm{~m}^{2}$ sur une période de onze ans. Ces deux approchent permettent de comparer ce que l'administration reconnaît comme étant déclaré (10 ha) et la réalité terrain (19 ha). La différence entre les deux valeurs est impressionnante puisque seulement $53 \%$ des remblais sont déclarés ce qui signifie que $47 \%$ de la redevance n'est pas perçue.

b) La consultation des données informatisées du GEGDP (Groupement d'Étude et de Gestion du Domaine public) du ministère de l'Équipement donne une valeur de $179603 \mathrm{~m}^{2}$ sur une période de seize ans entre 1986 et 2002 (résultats obtenues dans le cadre de l'AER (analyse éco régionale et non publiés). Ces dernières valeurs sont classées par demandeurs : territoire avec $48 \%$ des surfaces remblayées, hôtels avec $35 \%$, particuliers avec $15 \%$ et

TABLEAU 3. - Surfaces remblayées et cadastrées, affectées entre 2004 et 2014 (période couverte par le PGEM)

\begin{tabular}{|c|c|c|c|c|c|c|c|c|c|}
\hline Année & $\begin{array}{l}\mathrm{N}^{\circ} \\
\text { arrêté }\end{array}$ & Date & $\begin{array}{l}\text { Section de } \\
\text { commune }\end{array}$ & $\begin{array}{l}\text { Surface } \\
\mathrm{en} \mathrm{m}^{2}\end{array}$ & Prix en FCFP & $\begin{array}{c}\text { Coût } \\
\text { annuel }\end{array}$ & $\begin{array}{l}\text { Coût } \\
\text { au m }{ }^{2}\end{array}$ & Affectation & Remarque \\
\hline 2015 & 3368 & $23 / 04$ & Рaopao & $\begin{array}{c}\text { Déjà } \\
\text { comptabilisé }\end{array}$ & & gratuit & & TNAD & $\begin{array}{l}\text { Aménagement baie } \\
\text { de Paopao }\end{array}$ \\
\hline 2014 & 159 & $27 / 01$ & Afareaitu & 3623,00 & 94922600 & & $\begin{array}{c}26 \\
200\end{array}$ & Commune & $\begin{array}{l}\text { Aménagement } \\
\text { d'un accès public }\end{array}$ \\
\hline 2012 & 357 & $9 / 03$ & Paopao & 1181,00 & & 236200 & 200 & Privé & \\
\hline 2012 & 272 & $23 / 02$ & Paopao & 633,00 & & 126600 & 200 & SCI Rairoa & \\
\hline 2011 & 258 & $3 / 03$ & Haapiti & 127,00 & & 25800 & 203 & Privé & \\
\hline 2011 & 150 & $4 / 02$ & Раopao & 1181,00 & & 236200 & 200 & Privé & $\begin{array}{l}\text { Régularisation } \\
(2001\end{array}$ \\
\hline 2010 & 9068 & $17 / 12$ & Раopao & 1755,00 & & gratuit & & Equipement & $\begin{array}{l}\text { Extension du quai } \\
\text { de Maharepa }\end{array}$ \\
\hline 2010 & 1333 & $4 / 08$ & Раopao & 345,00 & & 69000 & 200 & Privé & $\begin{array}{l}\text { Régularisation } \\
(2005)\end{array}$ \\
\hline 2010 & 50 & $15 / 01$ & Afareaitu & 420,00 & & 84000 & 200 & Privé & $\begin{array}{l}\text { Régularisation } \\
\text { (2008) + rampe } \\
\text { d'accès pour } \\
\text { bateau } \\
\end{array}$ \\
\hline 2009 & 9481 & $30 / 12$ & Раopao & 76,00 & & 15200 & 200 & Privé & $\begin{array}{l}\text { Régularisation } \\
(2007)\end{array}$ \\
\hline 2009 & 1595 & $2 / 09$ & Papetoai & 106,00 & & 21200 & 200 & Privé & $\begin{array}{l}\text { Régularisation } \\
(2008)\end{array}$ \\
\hline 2009 & 162 & $30 / 01$ & Haapiti & 111,82 & & 16773 & 150 & $\begin{array}{l}\text { Hôtel les } \\
\text { Tipaniers }\end{array}$ & Ponton \\
\hline 2009 & 124 & $6 / 02$ & Рaopao & $\begin{array}{c}\text { Déjà } \\
\text { comptabilisé }\end{array}$ & & gratuit & & EAD & $\begin{array}{l}\text { Aménagement } \\
\text { dont institut de } \\
\text { la mer }\end{array}$ \\
\hline 2009 & 44 & $8 / 04$ & Teavaro & 51,00 & & 10200 & 200 & Privé & \begin{tabular}{|l} 
Régularisation \\
$(2007)$
\end{tabular} \\
\hline 2008 & 1976 & $26 / 12$ & Paopao & 2064,00 & & 412800 & 200 & Cook's Bay & Exploitation hôtel \\
\hline 2008 & 1975 & $26 / 12$ & Papetoai & 429,00 & & 85800 & 200 & Privé & Remblayé en partie \\
\hline 2008 & 1924 & $26 / 12$ & Paopao & 3350,00 & & & 0 & IJSPF & Zone de loisir \\
\hline 2008 & 192 & $6 / 02$ & Paopao & 114,00 & & 56600 & 496 & Privé & $\begin{array}{l}\text { Régularisation } \\
(2003)\end{array}$ \\
\hline 2008 & 163 & $30 / 01$ & Paopao & 137,00 & & 27400 & 200 & Privé & \\
\hline 2007 & 1515 & $9 / 11$ & Teavaro & 185,00 & & 37000 & 200 & Privé & \\
\hline 2005 & 636 & $19 / 08$ & Рaopao & $\begin{array}{l}\text { ND évalué à } \\
650\end{array}$ & & 129000 & & Privé & $\begin{array}{l}\text { Régularisation } \\
\text { d'une concession } \\
\text { maritime à charge } \\
\text { de remblai } \\
\end{array}$ \\
\hline 2005 & 404 & $18 / 02$ & Afareaitu & 189,00 & & 37800 & 200 & Privé & Régularisation \\
\hline 2005 & 401 & $27 / 06$ & Afareaitu & 563,00 & & gratuit & & $\begin{array}{l}\text { Direction de } \\
\text { la santé }\end{array}$ & \\
\hline & & & & 16640,82 & & 1628173 & & & \\
\hline
\end{tabular}


mairie, associations et divers avec $2 \%$. Les demandent des particuliers sont pour $40 \%$ des demandes de régularisation.

c) En consultant les arrêtés concernant les remblais à Moorea entre 2004 et 2014, nous notons que toutes les surfaces remblayées sont cadastrées.

La surface totale de $16109,82 \mathrm{~m}^{2}$ sera affectée soit à des organismes publics soit à des privés. Le tableau 3 indique l'année, le numéro de l'arrêté, la date dudit arrêté, la section de commune concernée, la surface en $\mathrm{m}^{2}$ concerne des remblais déjà effectués et cadastrés ainsi que l'affectation (privé, commune, société, etc.). Dans la liste des arrêtés, certains remblais cadastrés sont affectés à une personne ou une société plusieurs fois. C'est le cas des $2064 \mathrm{~m}^{2}$ accordés à l'hôtel Cook's Bay puis transférés à l'Etablissement public d'Aménagement et de Développement (EAD) suite à l'achat de l'ancien complexe hôtelier par ce dernier. Un nouveau transfert aura lieu en 2015, suite au changement de nom de l'Établissement public d'Aménagement et de Développement en l'Établissement public Tahiti Nui aménagement et développement (TNAD). À noter que cette affectation est destinée à l'aménagement de la baie de Paopao comprenant plusieurs projets dont un institut de la mer alliant formation et recherche en partenariat avec l'université de Berkeley et un site d'accueil des touristes de croisière.

Le Conseil économique, social et culturel (CESC), dans son rapport $\mathrm{n}^{\circ} 152$ de 2015, rapporte les valeurs suivantes :

«Moorea s'est dotée en 2004, d'un Plan de gestion de l'espace maritime (PGEM) qui prévoit l'interdiction des remblais sauf pour cause d'utilité publique. De 2004 à ce jour, 24 demandes de remblais ont été enregistrées, 21 ont été instruites; seules 3 étaient d'utilité publique."

Ces chiffres montrent que les habitudes résistent à la réglementation du PGEM, même si le nombre des demandes a diminué, mais aussi que ces demandes sont enregistrées par la direction des affaires foncières malgré la réglementation du PGEM, ce qui maintient la confusion. Le CESC conclut qu'une loi littorale équivalente de la loi métropolitaine faciliterait :
« la transversalité des problématiques liées au littoral dans plusieurs matières (aménagement, urbanisme, gestion du domaine public, environnement etc.), les outils de protection des rivages et du domaine public maritime du pays sont aujourd'hui traités de manière éparse. "

L'analyse qui précède sur les demandes et surfaces remblayées à Moorea permet les quelques conclusions suivantes. Au total près de $300000 \mathrm{~m}^{2}$ de zone frangeante du lagon ont été remblayées. Cette estimation est minimale car elle n'inclut pas les surfaces remblayées de façon illicites et non régularisées. A contrario, il est possible qu'une demande accordée ne soit pas exécutée mais ce cas est rarissime. En seize ans, de 1986 à 2004, la surface totale remblayée a été dix fois plus importante qu'après la mise en place du PGEM sur une période de dix années, 2004 à 2014. On peut donc en conclure, sous réserve des remblais non répertoriés, que le PGEM a été un frein à cette privatisation du lagon par les remblais littoraux.

Holstein (2011) a étudié les remblais le long de la ligne de rivage. Il note que les remblais publics représentent $11,4 \%$ du linéaire total de l'île contre $34,6 \%$ pour les remblais privés, soit un total de $46 \%$. Le tableau 4 indique le linéaire de remblais par section de commune en différenciant les remblais public et privés.

Suite à l'enquête menée auprès de la population, l'auteur note que :

« la question des remblais est délicate à aborder car de nombreux projets ont été construits sans aucune autorisation. [...] certains ont constitué leurs remblais depuis tant d'années qu'ils se pensent oublié des services". Il semblerait d'après cette étude que la principale raison donnée par les propriétaires à la construction d'un remblai littoral soit la protection $\mathrm{du}$ terrain contre les fortes houles (76\%), l'agrandissement du terrain $(12 \%)$, les raisons financières $(9 \%)$ et le reste la protection contre l'érosion. Au cours des entretiens, " la population tentait de justifier son appropriation du domaine public maritime par le passé tout en utilisant le terme - propriété qui est un terme utilisé uniquement à partir du $\mathrm{XIX}^{\mathrm{e}}$ siècle. » (Holstein, 2011 : 73)

Cet auteur précise aussi :

TABLEAU 4. - Linéaire de remblais à Moorea selon la catégorie et la section de commune (Holstein, 2011)

\begin{tabular}{|c|c|c|c|c|}
\hline $\begin{array}{c}\text { Section de } \\
\text { commune }\end{array}$ & $\begin{array}{c}\text { Linéaire remblais } \\
\text { privés en mètre }\end{array}$ & $\begin{array}{c}\text { Linéaire remblais } \\
\text { privés en } \%\end{array}$ & $\begin{array}{c}\text { Linéaire remblais } \\
\text { publics en mètre }\end{array}$ & $\begin{array}{c}\text { Linéaire remblais } \\
\text { publics en } \%\end{array}$ \\
\hline Afareaitu & 5300 & 7,4 & 700 & 1 \\
\hline Teavaro & 1895 & 2,5 & 2029 & 3 \\
\hline Paopao & 4415 & 6 & 1500 & 2 \\
\hline Papetoai & 4230 & 5,8 & 2000 & 3 \\
\hline Haapiti & 9045 & 12,6 & 209 & 3 \\
\hline
\end{tabular}


" il est aisé de constater que les remblais privés sont en plus grand nombre que les remblais d'utilité publique. Les concessions maritimes à charge de remblais sont majoritairement construites avec des enrochements dans le but de soutenir la terre remblayée. " (2011: 54)

Le propriétaire en bordure de lagon n'admet pas que la ligne de rivage soit mouvante et que, lors de forte houle, son terrain soit "grignoté" par dame Nature. Il doit donc le "protéger". Mais la nécessité de cette protection est souvent accentuée suite à l'extension de la propriété voisine vers cet espace communautaire privatisé. Ce privilège d'être en bordure de lagon devient un dû. Mais le rapport ne donne que des valeurs de linéaire et non de surface.

Notons que plusieurs études en sciences humaines ont été réalisées suite à l'adoption du PGEM de Moorea. Celle de Gaspar et Bambridge (2008) étudie les enjeux autour de la création d'aires marines protégées et aborde le statut traditionnel de l'environnement et celui de la gestion des ressources avant la colonisation. Les auteurs notent que sur le plan de la gestion foncière et maritime, les droits et les obligations se superposaient en quelque sorte en fonction des hiérarchies sociales. Cazalet (2008a, 2008b, 2009) et Féral (2008) étudient le droit des lagons de Polynésie française ainsi que le cadre juridique et institutionnel du Plan de Gestion de l'Espace Maritime (PGEM) de Moorea.

\section{L'impact écologique des remblais}

Rappelons que les zones remblayées se matérialisent en ligne de rivage par des remparts de blocs, volcaniques ou plus rarement coralliens et des murs en béton, au pied desquels arrive la mer à marée haute, parfois même à marée basse. De nombreuses recherches ont été menées sur Moorea et ont décrit en détail les impacts de la constructions des remblais sur l'écosystème récifolagonaire (Salvat, 1987; Galzin et al., 1989; Porcher, 1993). Ils peuvent se résumer ainsi :

- la destruction du récif frangeant,

- le risque d'eutrophisation,

- l'incidence sur la dynamique littorale,

- la pollution des eaux,

- la destruction de zones de décantation.

Le récif frangeant est par endroit une zone sablo-vaseuse parsemée de colonies coralliennes. C'est le lieu de recyclage de la matière organique et une zone de nourrissage pour les juvéniles de poissons de tout l'écosystème lagonaire.
La suppression de ce récif frangeant naturel pour "assainir la zone et la rendre plus jolie ${ }^{5}$ " conduit, à terme, à la diminution considérable des stocks de poissons adultes dans l'ensemble du lagon. Cette diminution a été suffisamment inquiétante pour que des études soient lancées en vue de mettre en place des récifs artificiels.

Les zones marécageuses, lieux de décantation des eaux pluviales chargées de terre, sont indispensables à la protection des coraux du voisinage. Leur disparition sous des remblais risque de se traduire par une forme de pollution diffuse incontrôlable et préjudiciable à la santé de l'ensemble du lagon.

De nombreuses études réalisées en Polynésie française mais également sur d'autres rivages tropicaux montrent sans ambiguiité que l'écosystème lagonaire est à la fois complexe et fragile et que toute modification effectuée d'une seule de ses parties sans étude sérieuse peut avoir des conséquences onéreuses et contraires à ce qui était voulu. Prenons l'exemple des murs construits en béton le long des remblais qui sont sensés les protéger contre l'érosion: ils amplifient la houle, ils jouent un rôle inverse en accentuant l'affouillement et donc l'érosion des zones sédimentaires frangeantes.

\section{L'impact économique des remblais}

Il est certain que la personne, qui réalise le remblai, aura la jouissance d'un terrain dont le prix sera plus faible que si elle avait dû l'acheter à un particulier. La réalisation d'un remblai est donc une véritable aubaine pour les propriétaires. Dans l'archipel de la Société, la surface remblayée autorisée est de 206 hectares dont 112 hectares pour les Îles-Du-Vent et 94 hectares pour les Îles-Sous-Le-Vent (AER, 2010). Sur 100 demandes d'occupation du domaine public maritime, 61 le sont par des particuliers et 15 par des promoteurs d'hôtels. Sur une période allant de 1993 à 2009, les îles les plus touchées sont par ordre d'importance: Tahiti avec $30 \%$ des demandes, Raiatea avec $23 \%$ et Bora-Bora avec $21 \%$ et pour Moorea seulement $5 \%$ suite à la mise en place du PGEM. Ces valeurs concernent une période allant de 1993 à 2009. Si nous prenons l'exemple de Moorea en 2015, un terrain au bord de l'eau peut coûter entre 10000 et $30000 \mathrm{FCFP}^{6} \mathrm{le} \mathrm{m}^{2}$ selon la localité, alors que le coût du remblai et de sa mise en place est de l'ordre de 3000 à 5000 FCFr le $\mathrm{m}^{2}$. Comme nous le verrons plus loin, la commune de Moorea a acheté au pays un remblai déclassé

5. Explication donnée couramment et curieusement par la direction de l'Équipement pour justifier la réalisation d'un remblai mais qui n'apparaît pas dans les motifs de la décision de concession car cela constituerait un motif évident d'annulation en cas de recours devant le tribunal administratif. 
26200 FCFr le m² en 2014 sur la côte est de l'île (section de commune d'Afareaitu : tableau 3).

En revanche pour la collectivité le problème se pose différemment. Tout d'abord une partie importante de la population se retrouve avec un accès restreint au lagon. Il y a privatisation de l'usage d'un espace communautaire que l'on doit pouvoir chiffrer. Ensuite, il y a le coût de la détérioration de la zone frangeante locale et de ses conséquences sur l'ensemble de l'écosystème lagonaire. Rappelons à titre d'exemple que la réhabilitation de la pointe Matira à Bora-Bora s'est chiffrée en dizaines de millions de FCFP suite à l'extraction de sable dans le lagon et par voie de conséquence une érosion de la pointe. Notons enfin que la création de récifs artificiels dont l'efficacité sera toujours inférieure à celle des récifs naturels est évaluée à environ 60 millions de FCFP pour un volume de $600 \mathrm{~m}^{3}$, volume qui correspond à un remblai modeste.

Une redevance est prévue à l'article 10 de la délibération $n^{\circ}$ 2004-34 APF du 12 février 2004 ainsi que les modalités de sa perception. Il est précisé que :

«L'autorité compétente tient compte, pour déterminer le montant des redevances dues, des avantages de toute nature procurés à l'occupant. "

L'exonération ou la réduction du paiement de la redevance sont abordés plus loin.

Bien que des études aient été réalisées dans le cadre du programme BEST, nous n'aborderons pas les valeurs des services écosystémiques c'està-dire des biens et services que les personnes peuvent tirer des écosystèmes, directement ou indirectement, pour assurer leur bienêtre même si le secrétaire d'État chargée de l'écologie affirmait en 2010 que ces évaluations économiques étaient nécessaires pour que l'environnement soit pris au sérieux. En effet, David et al. (2012) note que :

"au final, on a une valeur des services écosystémiques fournis par le récif mais cette valeur est erronée et son utilisation pour la gouvernance environnementale locale et l'aide à la décision opératoire n'est pas effective. "

\section{Les causes sociales des remblais}

La plaine littorale étant de surface réduite il peut sembler évident que l'occupation du domaine public maritime soit liée à l'évolution démographique. Dans l'étude concernant la commune d'Arue (Gros, 1995), bien qu'il y ait une corrélation entre la croissance démographique et l'augmentation du nombre de remblais entre 1963 et 1971 , les deux phénomènes conjoints ont eu une cause extérieure. Elle correspond à l'installation du CEP qui entraîne un déferlement des populations à Tahiti et donc des besoins nouveaux en logement, équipements, élargissement des routes, etc. Sont liés à ce chamboulement un apport financier très important et l'arrivée massive d'engins de terrassement. Pour bénéficier d'une image positive auprès de la population, l'État a accédé volontiers aux vœux des habitants, car à cette époque, c'est le gouverneur qui est le chef de l'administration et donc du service de l'équipement. Dans l'étude sur les Îles-Sous-Le-Vent (Marquet, 1994), l'auteur arrive à une conclusion similaire, à savoir qu'il n'y a pas de lien direct entre l'importance de la population et le taux d'anthropisation du littoral. Il constate également que l'anthropisation du rivage se fait en général au détriment de la végétation haute. La détermination d'une catégorie de ligne de rivage en un point donné est le résultat de l'interaction de facteurs naturels et humains, ces facteurs humains étant à la fois sociaux, économiques et culturels. L'auteur précise que la majorité des concessionnaires n'a aucune notion des dégâts occasionnés au milieu naturel. Une partie de ces études sont reprises dans le rapport du CESC de 2013.

A noter que la non application des articles 36 et 39 de la délibération $\mathrm{n}^{\circ}$ 2004-34 APF du 12 février 2004 concernant la servitude de passage des 3 mètres qui n'est jamais respectée ni contrôlée et imposée par l'administration est une incitation à la réalisation de remblais car le bénéficiaire de la concession a "les pieds dans l'eau » sans être incommodé par la présence d'un passage public. L'occupation exclusive (de droit) d'une portion du domaine public se double d'une privatisation (de fait) de l'accès à la mer sur un espace normalement public, inaliénable et imprescriptible.

\section{Le rôle des services administratifs et leurs limites}

Actuellement, la procédure d'instruction et de recevabilité des demandes d'occupation de dépendances du domaine public est régi par l'arrêté $n^{\circ}$ 385 сM du 4 mars 2004 dont la dernière modification date du 31 décembre 2007. Elle définit :

- la recevabilité de la demande,

- son instruction,

- la délivrance ou refus d'autorisation,

- les redevances.

L'administration compétente pour instruire les demandes de concessions maritimes à charge de remblais est la direction des Affaires foncières. Dans tous les cas, l'instruction du dossier comporte la consultation du maire de la

6. NDLR. -1000 FCFP $=8,38 €$. 
commune qui reçoit un exemplaire du dossier envoyé par la direction des Affaires foncières. Pour toutes consultations et en l'absence de réponse dans un délai d'un mois, l'avis est réputé favorable (modification apportée par l'arrêté $\mathrm{n}^{\circ} 1942 \mathrm{~cm}$ du 28 décembre 2007).

La commission du domaine public est composée de membres à voix délibératives et de membres à voix consultatives. En mars 2004, les membres à voix délibérative sont les ministres en charge du Domaine (président de la commission), de l'Équipement(vice-président), del'Environnement et du Tourisme, ainsi que le maire et le chef de la circonscription administrative territoriale du lieu concerné. Les services du Tourisme et de la Pêche ont des voix consultatives. En 2007, les ministres en charge de l'Environnement et du Tourisme qui avaient voix délibératives ne sont plus membres de la commission de même que le maire de la commune qui peut toutefois assister à la séance à titre consultatif et à sa demande. Disparaissent également de la liste des membres à voix consultative le service du Tourisme et celui de la Pêche. Cette modification est importante car elle donne un rôle prépondérant aux deux seuls ministres des Affaires foncières et de l'Équipement à voix délibérative dans la formation de la décision. Les autorisations d'occupation temporaire du domaine public sont accordées par arrêté pris en conseil des ministres, sur le rapport du ministre en charge des Affaires foncières président de la commission qui rend compte de l'avis de la commission.

Il n'est pas rare de voir un membre du Cabinet d'un ministre le remplacer, alors qu'en toute logique ce dernier ne devrait être remplacé que par un autre ministre.

Toute demande de renouvellement est effectuée six mois au moins avant la date d'expiration de l'autorisation. Cette demande est adressée par simple lettre au service compétent pour recevoir la demande d'autorisation d'occupation.

Au fur età mesure de la réalisation de ces différents remblais, les communes ont demandé des «Plans d'alignement " qui correspondent à un nouveau dessin de la ligne de rivage et toujours avec une partie de récif frangeant à remblayer. Même si certains de ces plans d'alignement ont reçu un avis favorable de la CSMN et ont été approuvés en conseil des ministres, ils n'ont aucune base juridique et aucune étude n'a participé à leur tracé. De plus de "plans d'alignement" en "plans d'alignement" les surfaces remblayées s'élargissent et couvrent des surfaces de récifs frangeant de plus en plus importantes. Cela peut conduire à la disparition du récif frangeant et donc des fonctions indispensables à l'équilibre de l'ensemble de l'écosystème.

Malgré la réglementation mise en place et la création de commissions, il est apparu difficile voire impossible de supprimer tout remblai illicite. En effet, la procédure courante pour réaliser un remblai sans autorisation est toujours la même. Le remblai est construit à la vue de tous. Cette réalisation est suivie d'une demande de régularisation qui même si elle est refusée ne conduit pas à une remise en état systématique des lieux par le contrevenant. Pourtant si les sanctions du chapitre III de la délibération $\mathrm{n}^{\circ}$ 2004-34 ne prévoient que des contraventions de grande voirie (article L774-1 et suivants du code de justice administrative), c'està-dire des peines correspondent à la cinquième classe du code pénal, l'auteur de la contravention " pourra être tenu de réparer le dommage causé, au besoin sous astreinte".

Il apparaît que sur 185 dossiers traités, la CSMN a donné un avis favorable dans $32 \%$ des cas. Sur les 120 dossiers ayant reçu un avis défavorable, 28 ont été autorisés par le conseil des ministres et $60 \%$ d'entre eux concernaient des demandes provenant de particuliers.

Il apparaît également que le conseil des ministres ne se prononce que sur la moitié des dossiers. Quant on sait que la plupart sont des demandes de régularisation, il y a de quoi être inquiet quant au manque à gagner par défaut de redevance d'occupation. Ce manque à gagner a été estimé à 46 millions de FCPF sur 5 ans. Il s'agit essentiellement de remblais appartenant à des particuliers qui jouissent gratuitement de terrains appartenant à la collectivité.

Les demandes cumulées depuis 1952, conduisent à une surface officielle occupée du DPM d'environ 700 ha. Les équipements publics (quais) et les concessions accordées à des hôtels (bungalows sur l'eau) sont les plus grands consommateurs d'espace. À titre d'exemple, depuis 1975, 15 demandes intéressent le secteur hôtelier à Bora-Bora pour une surface totale de $431922 \mathrm{~m}^{2}$ (avec un maximum de $140000 \mathrm{~m}^{2}$ pour un hôtel), ( $c f$. AER Polynésie française - synthèse des connaissances - usages et pressions). En 2008, par exemple, 354 dossiers ont été instruits: 219 à charge de remblais, 45 demandes d'implantations de pontons, 19 d'occupations de lais de mer, 33 pour des projets hôteliers (bungalows sur l'eau et autres) et 58 divers (mouillages, émissaires, déclassement DPM, occupation de la zone des 50 pas aux Marquises etc.). Depuis 1952, $60 \%$ des demandes concernent des concessions à charge de remblais et $30 \%$ des pontons ou wharfs (CESC, 2015).

Un bilan présenté en 2013, indique que 101 procès ont été gagnés par le pays et 3 remises en état des lieux ont été effectuées avec un manque évident de publicité qui aurait pu avoir un effet dissuasif, mais la période couverte n'est pas indiquée. Il est vrai que quand un remblai a été réalisé, il a modifié le milieu lagonaire de façon définitive et la remise en état va perturber pour la seconde fois ce milieu. Donc il peut paraître préférable de ne rien toucher, mais si le but est de faire un exemple, l'approche doit être différente. 
La définition des objectifs est donc essentielle.

Concernant l'efficacité du PGEM de Moorea, Cazalet (2008a) note :

" À titre d'exemple significatif, le règlement du PGEM l'autorise [le comité permanent] à donner son avis ("être entendu") lors de "l'instruction des demandes d'occupation du domaine public maritime". Il s'agit d'un avis simple certes, mais le plus souvent négatif et systématiquement suivi par le ou les ministres délivrant l'autorisation. Ceci mérite d'être relevé, alors même que les avis négatifs des commissions obligatoires en la matière sont en général ignorés, à la grande frustration des administrations territoriales responsables de l'instruction. "

\section{La politique du gouvernement}

À la lecture des délibérations, il apparaît que les concessions maritimes, à charge de remblais, définitives, deviennent temporaires à partir de la délibération n ${ }^{\circ} 78-128$ du 3 août 1978. Cette évolution est importante. Elle correspond surtout à la mise en conformité avec la loi domaniale car même si le milieu a été modifié par les remblais, le pays confirme qu'il reste propriétaire de cet espace d'une manière imprescriptible puisque l'article 7 de la délibération $n^{\circ}$ 2004-34 APF du 12 février 2004 précise que :

"les autorisations d'occupation d'une dépendance su domaine public sont délivrées à titre personnel et précaire. Elles sont révocables à tout moment ».

\section{La redevance}

La redevance pourrait donc être assimilée à un loyer. Mais cette notion de location nécessite quelques remarques.

1. L'évaluation par le pays du loyer annuel d'un terrain nu est de l'ordre de $5 \%$ de sa valeur marchande. Dans le cas de Moorea par exemple, le terrain en bord de mer étant estimé entre 20000 et 30000 FCPF le $\mathrm{m}^{2}$, le montant du loyer annuel serait compris entre 1000 et $1500 \mathrm{FCPF} / \mathrm{m}^{2}$. Or nous avons vu qu'il est de $200 \mathrm{FCPF} / \mathrm{m}^{2}$ soit de 5 à 7,5 fois moins cher.

2. Le montant de la redevance est fixé par le conseil des ministres. Elle est identique quelque soit le lieu ou l'île concernée, ce qui n'est pas le cas de la valeur d'un terrain en bord de mer. Cette redevance ne peut donc pas être considérée comme un loyer.

3. L'espace terrestre gagné sur le domaine maritime n'est pas construit pas le propriétaire (le pays) mais par le locataire des lieux. Enfin chaque délibération confirme que la personne qui bénéficie de la concession maritime doit conserver de manière effective le passage public de 3 mètres de largeur le long de sa façade maritime. Il serait intéressant de connaître les critères qui permettent de calculer la redevance et les raisons de cette uniformité du coût quel que soit le lieu et l'activité réalisée.

\section{Le passage public}

Les délibérations qui se sont succédées depuis 1978 prévoient un passage public de 3 mètres de largeur le long de sa façade maritime maintenu praticable par le titulaire pour les piétons. Cette servitude est par définition inaliénable ${ }^{7}$. Même si à certaines époques, le pays a voulu protéger la ligne de rivage par la suspension des autorisations d'occupation temporaire du domaine public maritime, à aucun moment l'article 36 de la délibération n'a été appliqué. De plus sa non application ne semble avoir ému personne.

\section{Les suspensions d'autorisations}

Le 23 novembre 2010, sous le gouvernement de Gaston Tong Sang, l'arrêté n' 2121 pris en conseil des ministres suspend provisoirement la délivrance des autorisations d'occupation temporaire du domaine public maritime pour une durée d'un an. Tearii Alpha est ministre des Affaires foncières, de l'Aménagement, de l'Habitat et de l'Équipement. Le 7 novembre 2011, l'arrêté précédent est prorogé d'un an.

Le 27 décembre 2012, sous le gouvernement Temaru, l'arrêté $\mathrm{n}^{\circ} 2152$ pris en conseil des ministres suspend pour une durée de deux ans la délivrance des autorisations d'occupation temporaire du domaine public maritime. Cet arrêté concerne la réalisation de remblais, l'occupation de dépendances remblayées, l'occupation du rivage, de lais et de relais de mer, la réalisation d'un chenal. Une dérogation est prévue pour les activités touristiques commerciales et industrielles sous réserve que l'impact sur l'environnement soit maitrisé et que l'occupation soit proportionnée à l'activité exercée et qu'elle en constitue un accessoire.

\section{Un retour en arrière}

Le 22 janvier 2015, soit un mois après la fin de la suspension des autorisations, la presse titre : "Le conseil économique, social et culturel se déchire sur les remblais illégaux». En effet, le gouvernement Fritch avait soumis au CESC un projet de loi de Pays visant à régulariser les remblais illégaux. L’article de ce projet de Loi de Pays précisait que : 
" cette régularisation concernerait environ 360 remblais et irait jusqu'à permettre aux contrevenants de racheter ces terres. »

Or la législation actuelle ne prévoit le rachat des terres remblayées que dans des conditions précisées à l'article 39 de la délibération nº 2004$34 \mathrm{APF}$ du 12 février 2004.

"Cette aliénation n'est possible qu'au profit des particuliers et établissements ayant une activité économique à caractère permanent, occupant le domaine public déclassé etc. "

"Je dis qu'on ne peut pas régulariser des infractions comme ça. Tel que l'article 39-1 est écrit, il équivaut à donner l'absolution à des délinquants quatre fois en infraction : pour le remblai illégal, le non-paiement des redevances pour le remblai, la construction d'une maison sans permis de construire et enfin le nonpaiement de l'impôt foncier sur cette maison. Je ne peux pas accepter cette politique du fait accompli !»

s'est exclamé lors des débats Éthode Rey. Le texte a tout de même été validé par le CESC avec 16 voix pour, 13 contres et 5 abstentions.

Le rapport $n^{\circ} 152$ section "Aménagement" du 21 janvier 2015 rédigé par deux membres du CESC, Patick Galenon et Winiki Sage (opposés au projet de Loi de Pays) sur l'avenir de la Polynésie française face à une gouvernance durable de son patrimoine marin :

" veut mettre l'accent sur la manière dont peuvent s'établir et se consolider les relations entres ces différents acteurs en vue d'aboutir à une gestion appropriée et équitable des différents secteurs liés à la mer ".

Il est difficile d'imaginer que cette loi puisse résoudre le problème des remblais illicites, pas plus que ne l'ont fait les plans d'alignement. Au moment où l'on parle de changement climatique, de la montée des eaux et donc du devenir du littoral, où l'élaboration des plans de prévention des risques est en souffrance et où la sécurité des personnes devrait être une préoccupation, la position du gouvernement laisse perplexe. À noter dans le même temps la révision du code de l'aménagement afin de modifier la procédure des PPR (Plan de prévention des risques) pour une gestion plus pragmatique et plus souple des PPR. La presse du 21 juillet 2015 indique :

«Le projet de texte vise principalement à mettre en place une procédure simplifiée pour lever ou préciser un risque dans un plan de prévention des risques (PPR), notamment dans le cadre de PPR dits approuvés, et a intégrer, au sein de cette procédure simplifiée, une modalité d'information du public. Actuellement, seule la commune de Punaauia dispose d'un PPR approuvé, les PPR des autres communes demeurant au stade de projet ».
Concernant les remblais construits sans autorisation par exemple, la procédure pour leur remise en état a évidemment été confirmée par l'autorité judiciaire, et ce sans effet. Ce problème de l'application des textes réglementaires et des décisions de justice mérite que l'on s'y arrête ; et si une évaluation doit être réalisée c'est à ce niveau que la priorité doit être donnée et non dans l'élaboration de nouveaux textes.

Nous ne pouvons pas imaginer que la politique du gouvernement soit inexistante ou qu'il n'y ait aucun lien dans l'approche de cette politique entre le changement climatique et les risques qui sont liés à la montée du niveau de la mer, à l'augmentation de l'intensité des cyclones d'un côté et à l'aménagement du littoral de l'autre côté. Nous pouvons seulement dire que nous n'y avons pas eu accès.

\section{Conclusion}

Rappelons que notre propos était d'analyser la réglementation et son application concernant les concessions maritimes à charge de remblais en zone frangeante du lagon. Il ne concernait pas les concessions maritimes permettant d'utiliser un volume d'eau aux fins d'élevage ichtyologique ou d'huîtres perlières. Concernant les demandes de concessions à charge de remblais on peut distinguer trois motivations principales dont les objectifs et les demandeurs sont nettement différents :

1. Les aménagements d'intérêt public par le Pays ou les mairies,

2. Les besoins liés à l'industrie touristique par un hôtelier,

3. L'intérêt particulier d'un propriétaire possédant une propriété en bordure du lagon et qui veut l'agrandir.

Les deux premières motivations s'inscrivent dans le cadre du développement du pays. En effet, certains remblais comme la construction et les agrandissements successifs du Port de Papeete, ou encore la construction de l'aérodrome de Faaa, ont été nécessaires, voire indispensable. Mais quand il s'agit de remblais recevant des écoles comme à Arue ou la caserne des Pompiers à Punaauia, on peut mettre en balance la nécessaire protection du lagon et l'inaliénabilité du domaine maritime dû à un aménagement de la plaine littorale peu ou mal pensé, sans vue d'ensemble alors qu'il existe une procédure pour élaborer des PGA dans le cadre de SAGE (Schéma d'aménagement général) ${ }^{8}$. 
Le politique est confronté à cette double problématique de développement, d'une part, et de protection de l'environnement, d'autre part. Les anciennes décisions d'autorisation de remblais pour cause d'intérêt public sont devenues des dérogations à des textes en faveur du respect de l'environnement. Certaines zones remblayées sont voulues par l'administration sur décision politique comme la réalisation du centre de loisir ou les jardins de Paofai à Tahiti.

Pour la troisième motivation, celle des particuliers agrandissant leur propriété en bord de plage, les politiques n'ont aucune excuse à enfreindre les lois qu'ils ont eux même adoptées. Les autorisations de concession à charge de remblais par des particuliers obéissent à des considérations moins électoralistes que liées aux privilèges des "grandes familles" et à un laxisme politique que rien ne justifie, en tous les cas pas l'intérêt public. On note toutefois une volonté politique à certains moments de se ressaisir sur ce sujet : concessions définitives devenues temporaires, suspensions à plusieurs reprises de toute autorisation, demandes plus rares de dérogation. En revanche l'acceptation en elle même d'une procédure de "régularisation " est la porte ouverte à toute incitation de remblai auto décidée alors que le remblai sauvage et illicite devait être suivi d'une action en justice avec remise en état des lieux. Enfin le droit de passage non respecté est un déni de citoyenneté accepté politiquement au mépris des habitants et de leur cadre de vie. Sans compter que l'attention portée à la pêche de subsistance ou artisanale, pourtant culturellement et socialement importante avec son rôle de "stabilisateur social " est insuffisamment prise en compte. Enfin, l'absence d'une base de données gérée par ou à la demande du pays accentue la méconnaissance de l'état des lieux du littoral et de sa privatisation et donc l'impossibilité de mener une politique volontariste sur ce sujet, si toutefois le politique le veut (Aubanel, 2013).

En conclusion, on peut noter que la gestion de ces concessions à charge de remblais s'améliore au fil du temps. Il est certain que les choses évolueront. C'est au politique d'affirmer ses options fondamentales pour le développement du Pays. Ces options concernent les choix de dérogation pour remblaiement d'intérêt public qui devront être de plus en plus judicieux. Ces options sont celles de conserver au littoral un paysage naturel apprécié par la collectivité des résidents et des touristes et respectueux de l'inaliénabilité du domaine public maritime et du droit de passage en bord de mer. Conserver le patrimoine naturel qui fait la richesse du Pays, et pérenniser le fonctionnement des écosystèmes qui le constitue, est une nécessité vitale pour l'avenir.

Ces considérations sur la politique menée et à mener concernant les remblais se doublent actuellement d'une préoccupation majeure relative au trait de côte et au littoral relativement aux prédictions du changement climatique. Cette préoccupation est mondiale, nationale mais elle place les petits pays insulaires océaniens au premier plan. La protection qu'assurent actuellement les remparts de blocs basaltiques ou coralliens de même que les constructions cimentées qui bordent les zones remblayées, résisteront elles à une élévation du niveau de l'océan et des lagons de $90 \mathrm{~cm}$ à l'horizon 2100 (Church et al., 2013 ; Salvat, 2015) ? À noter qu'il s'agit là du pire des scénarios suite aux comportements de l'humanité vis à vis des rejets de gaz à effets de serre. La question des remblais et leur fonctionnalité dans cette perspective d'élévation du niveau de la mer n'est pas particulière mais concerne l'ensemble de la ligne de rivage naturelle ou anthropisée. En Polynésie française, les autorités se sont saisies de ces questions depuis plusieurs années avec la mise en place de Plan de prévention des risques naturels (PPR) qui se sont concentrés sur les effets des tsunamis, des cyclones, des inondations et des précipitations abondantes pouvant engendrer des glissements de terrain, programmes ARAI depuis 2000 avec le BRGM en liaison avec le service de l'Urbanisme (Lecacheux et al., 2013 ; CREOCEAN, 2015). En décembre 2011, une conférence régionale s'est tenue à Papeete sur les politiques d'aménagement du littoral dans une perspective d'adaptation au changement climatique (colloque régional sur les politiques d'aménagement du littoral dans une perspective d'adaptation au changement climatique, 2011). Plus récemment, en juin 2015, chercheurs et autorités ont tenu un colloque sur « la vulnérabilité des îles basses polynésiennes et du Pacifique face aux effets du changement climatique " et des programmes de recherches ont été lancés sur la vulnérabilité des îles, tout particulièrement des atolls des Tuamotu comme STORISK - petites îles face au changement climatique, échelles de risques et adaptation - (Duvat et Salvat, à paraître). Ces problèmes amènent une réflexion sur le changement des conditions de vie et de cadre de vie sur lesquels le Pays devra réfléchir comme ses voisins insulaires du Pacifique sud (Louis Savoie, comm. pers.). Rappelons que la COP 21 vient d'approuver un protocole d'accord et que les Pays océaniens dont la Polynésie française avec son Plan Climat ont un rôle à jouer, pas seulement comme victime mais comme force de proposition. C'est dans ce contexte que la politique publique des gouvernements à venir sur les concessions à charge de remblais doit être imaginée. Faut-il attendre que le pouvoir politique en ait pleinement conscience et prenne les décisions qui ne seront pas forcément populaires au niveau de l'individu mais qui correspondront à une réelle politique publique du littoral qui donnera une unité, le fameux "vivre ensemble » indispensable à la cohésion sociale. 


\section{Remerciements}

Je remercie Bertrand Cazalet, François Feral et Bernard Salvat pour les discussions qui ont permis d'enrichir cette analyse, ainsi qu'un relecteur du comité de rédaction pour ses remarques. Cet article s'inscrit dans le cadre du programme BEST (Biodiversité, écosystèmes, services écosystémiques, territoires d'Outre-Mer européens) et du programme RESCCUE (Restauration des services écosystémiques et adaptation au changement climatiques).

\section{BIBLIOGRAPHIE}

AER, 2010. Analyse Éco-Régionale marine de Polynésie française. Synthèse des connaissances usages et pressions, Polynésie française, Agence des Aires Marines Protégées.

Aubanel Annie, 1993a. Valeurs socio-économiques du milieu corallien et de ses ressources, application à une île océanique du Pacifique Sud : Moorea, archipel de la Société, thèse de doctorat, Géographie tropicale et aménagement de l'Université Michel de Montaigne, Bordeaux III, $344 \mathrm{p}$.

—, 1993b. Procédures et pratiques des concessions maritimes à charges de remblai : motivations et conséquences écologiques et économiques, le cas de Moorea, Les écosystèmes, actes des troisièmes journées de la recherche en Polynésie française du 27 au 28 octobre 1993, pp. 41-47.

—, 2013. Éléments de réflexion sur la politique d'aménagement du littoral entrant dans la composition du schéma d'aménagement général (SAGE), Service de l'aménagement et de l'urbanisme, $15 \mathrm{p}+$ annexes.

Bambridge Tamatoa et Jacques Vernaudon, 2013. Une appropriation foncière de l'espace terrestre et marin, in Étienne Le Roy, La Terre et l'homme. Espace et ressources convoitées entre le local et le global, actes du congrès d'ISAIDATSIRD, Paris, Karthala, pp 33-71.

Benet Agnès, 2009. Évolution des zones côtières en milieu insulaire et impact du changement global : Perspectives 2100, thèse de doctorat université de Polynésie française 243 p.

Cazalet Bertrand, 2008a. Historique, cadre juridique et institutionnel du Plan de gestion de l'espace maritime (PGEM) de Moorea, Polynésie française, projet GAIUS/WP2/01, juin 2008, $47 \mathrm{p}$.
Cazalet Bertrand, 2008b. Droit des lagons de Polynésie française, Revue juridique de l'Environnement (RJE), 4, pp. 391-407.

Cazalet Bertrand, 2009. La protection des lagons de Polynésie française : enjeux politiques et incertitudes juridiques, The 11th Pacific Science Intercongress, 02-06 mars 2009, Tahiti.

CESC, 2013. L'aménagement des plages publiques en Polynésie française: entre une indispensable exploitation et valorisation touristique, rapporteurs Pascal Luciani et Toni Tereino, rapport $n^{\circ} 151 /$ CESC adopté en assemblée plénière le 31 octobre 2013, $74 \mathrm{p}$.

—, 2015. L'avenir de la Polynésie française face à une gouvernance durable de son patrimoine marin, rapporteurs Patrick GALENON et Winiki SAGE, rapport $n^{\circ} 152 /$ CESC adopté en assemblée plénière le 21 janvier 2015, 146 p.

Church J.A. et al., 2013. Sea Level Change, in Stocker, T.F., D. Qin, G.-K. Plattner, M. Tignor, S.K. Allen, J. Boschung, A. Nauels, Y. Xia, V. Bex and P.M. Midgley (eds), Climate Change 2013: The Physical Science Basis, contribution of Working Group I to the Fifth Assessment Report of the Intergovernmental Panel on Climate Change, Cambridge, United Kingdom and New York, NY, USA, Cambridge University Press.

Colloque Régional sur les politiques d'aménagement du littoral dans une perspective d'adaptation au changement climatique, 2011. Ministre de l'aménagement et du logement en charge des affaires foncières et de l'urbanisme Tahiti, décembre 2011, 75 p.

CREOCEAN, 2015. État de l'environnement en Polynésie française 2007/2014, document coordonné par F. SÉGuIN et réalisé en étroite collaboration avec Catherine Gabrie, Emmanuelle Gindre, Fred Jace, T. Ramage, Clive Wilkinson, CRIOBE, Bureau de Veille Sanitaire de la Santé (Bvs), Tahiti, Direction de l'environnement.

David Gilbert, Émilie Mirault, Gwenaëlle Pennober et Christophe Révillion, 2012. Unités paysagères et services écosystémiques, l'exemple des récifs coralliens, Vertigo, la revue électronique en sciences de l'environnement, hors-série $14,15 \mathrm{p}$.

Duvat Virginie et Camille Salmon, 2015. Étude de l'évolution de la position du trait de côte des îles coralliennes des atolls de Rangiroa et de Tikehau, projet REOMER 2013-2016, 28 p. + annexes. 
Duvat Virginie et Bernard Salvat, (à paraître). Drivers of shoreline change in Pacific atolls reef islands, en cours de soumission.

FÉral François, 2008. Rapport de synthèse sur la mission gaIUs en Polynésie française, GAIUS/WP I/02, $29 \mathrm{p}$.

Galzin René, Catherine Gabrié, Anne Lefèvre et Bernard Salvat, 1989. Dégradations anthropiques des zones frangeantes des récifs coralliens polynésiens, influences sur l'écosystème, incidences sur les peuplements ichtyologiques, SRETIE, rapport antenne EPHE/Muséum, $52 \mathrm{p}$.

Gaspar Cécile et Tamatoa Bambridge, 2008. Territorialités et aires marines protégées à Moorea (Polynésie française), Journal de la Société des Océanistes 126-127 : Spécial Environnement dans le Pacifique (J. Trichet et I. Leblic éds), pp. 231-246 (http://jso.revues. org/2462).

Gros Sophie, 1995. Les remblais ; étude des motivations, procédures et réalisations, l'exemple d'Arue (Tahiti, Polynésie française). Université française du Pacifique, DEA Connaissance et gestion des milieux coralliens littoraux et océaniques, $33 \mathrm{p}$.

Holstein Alexia, 2011. Les remblais sur la ligne de rivage de Moorea (Polynésie française), Université de Rennes 2 / CRIOBE, $88 \mathrm{p}+$ annexes.

Lecacheux S., T. Bulteau, R. Pedreros, E. Delvallée et F. Paris, 2013. arais 3 : Evaluation probabiliste des houles et des surcotes cycloniques en Polynésie française, rapport final BRGM/RP-6I888-FR, $120 \mathrm{p}$.

Lechat Philippe, 1976. Les concessions définitives du domaine public maritime accordées depuis 1956 en Polynésie française : étude quantitative, service de l'Aménagement et de l'Urbanisme, $42 \mathrm{p}$.

Leille Luana, 2005. Caractérisation de la ligne de rivage de l'île de Tahiti, Polynésie française, EPHE, Naturalia et Biologia, IFreCor Polynésie : RA 137, 20 p. + annexe $\mathrm{n}^{\circ} 1$ cartographie du trait de côte de l'île de Moorea, annexe $\mathrm{n}^{\circ} 2$ cartographie des différentes catégories de la ligne de rivage de l'île de Tahiti au 1/5000 (jeu de 90 supports plans).

Lison DE Loma Thierry, 2007. Catégorisation de la ligne de rivage des communes de Tahaa et d'Uturoa (îles sous le Vent), demande de la direction des Ressources marines, $10 \mathrm{p}$.

Marquet Nicolas, 1994. La ligne de rivage des Îles-Sous-Le-Vent (Archipel de la Société, Polynésie française). Catégorisation et Quan- tification, taux d'anthropisation, Université française du Pacifique, DEA Connaissance et gestion des milieux coralliens littoraux et océaniques, 31 p. + annexes.

MEDDE, 2015. Rapport final État des lieux Mer et Littoral présenté au ministre de l'Ecologie, du Développement durable et de l'Energie, $340 \mathrm{p}$.

Paskoff Roland, 1993. Côtes en danger, Paris, Masson éd., coll. Pratique de la Géographie.

Polti Sandrine, 2001. Caractéristique de la ligne de rivage et du domaine maritime de l'île de Moorea, Polynésie française, CRIOBE, EPHE, IFreCor Polynésie : RA 97, 28 p. + annexes.

Porcher Michel, 1993. Intertropical coastal and coral reef areas and their development. Pratical guide, study methodologies, technical recommandations, ministère de l'Environnement, direction de la Nature et des Paysages, 238 p.

Raynal Jean-Baptiste, 2004. Le littoral de Taiarapu Ouest, un espace en mutation ?, mémoire de maitrise de l'Université de la Polynésie française, Paris IV Sorbonne.

Roucaute Jules, 1953. Etude sur la situation juridique, dans les EFO, des dépendances naturelles du domaine public telles qu'elles sont énumérées dans l'article 558 du code civil (et plus particulièrement les rivages et les eaux du lagon et de la mer, ainsi que les récifs), Etablissement français de l'Océanie, service des domaines, $18 \mathrm{p}$.

SaLVAT Bernard, 1987. Human impacts on coral reefs: facts and recommandations, Antenne de Tahti EPHE/museum, Polynesie française.

—, 2015. Health and Degradation of Coral Reefs: Assessment and the Future, in Ceccaldi et al. ed., Marine Productivity: Perturbations and Resilience of Socio-ecosystems, Springer International Publishing, pp. 343-354.

Vieux Caroline, Yannick Chancerelle, Annie Aubanel et Bernard Salvat, 2008. Les modifications de la ligne de rivage dans les îles de la Société (Polynésie française) : un indicateur des pressions anthropiques en zone côtière, Journal de la Société des Océanistes 126-127: Spécial Environnement dans le Pacifique (J. Trichet et I. Leblic éds), pp. 59-66 (http:// jso.revues.org/3162).

Villot René, 1990. La conservation du domaine public territorial, ministère de l'Équipement, direction de l'Équipement, groupement études et gestion du domaine public, 69 p. + annexes. 

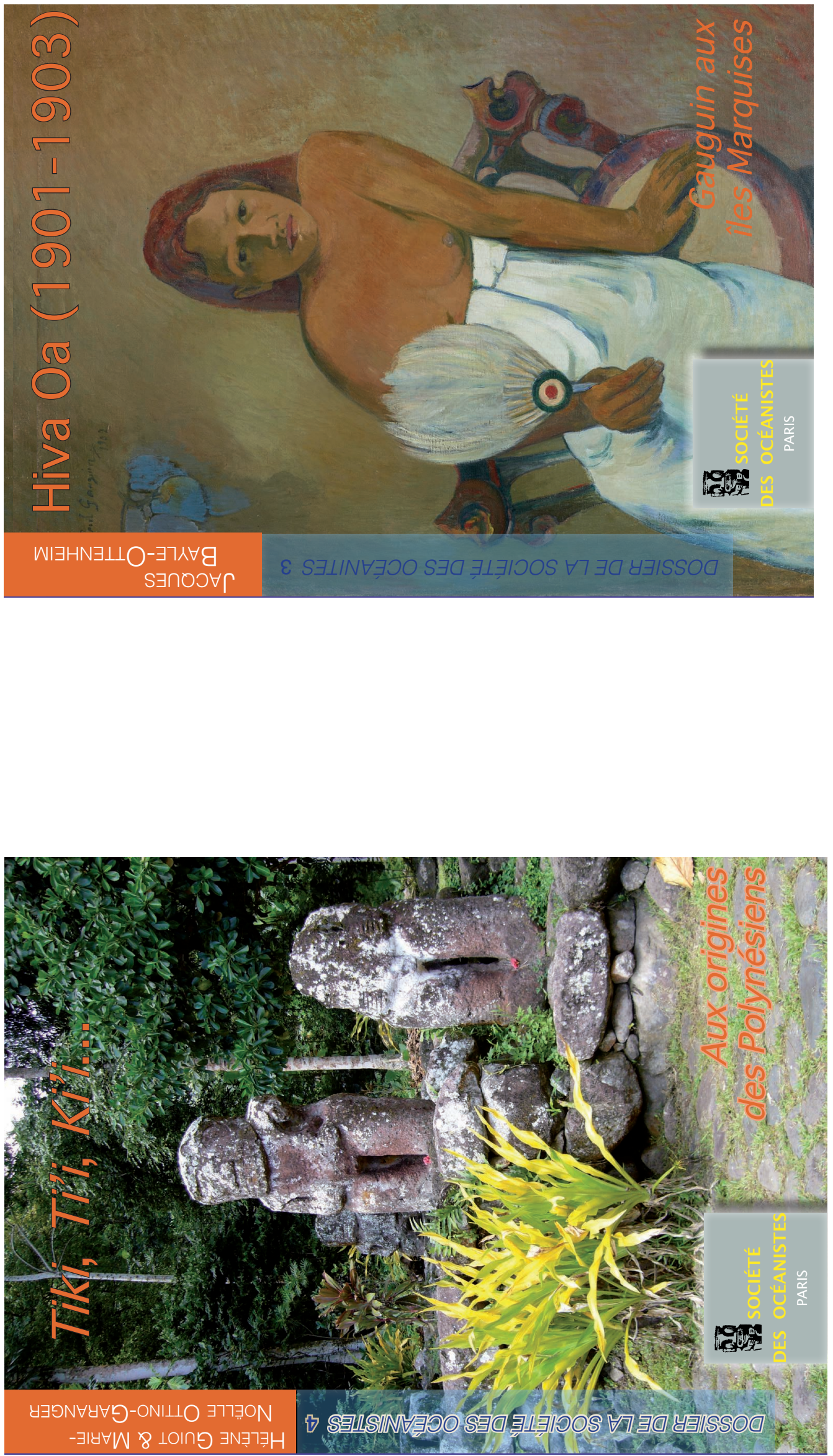